\title{
Human Capital and Wage Determination in Different Ownerships, 1989-97
}

\author{
Xing Chunbing*
}

October 2006

\begin{abstract}
This article evaluates the Chinese labour market by examining the role of human capital in wage determination. Using data from China's Household Nutrition Survey, we estimate the returns to education in state-owned, collective-owned and private sectors. In the private sectors, the returns to education increase from 0-2 per cent in the late 1980s and early 1990s to about 4 per cent in 1997, while those of public sectors do not increase. This suggests that the labour market is segmented and human capital is more explicitly rewarded in the private sector. We correct for self-selection error using switching model, but the main results remain unchanged.
\end{abstract}

Keywords: labour market segmentation, human capital, wage determination, transition

JEL classification: J42, J24, J31, P23

Copyright @ C UNU-WIDER 2006

* School of Economics and Business Administration, Beijing Normal University, email: xingchunbing@gsm.pku.edu.cn

This study has been prepared within the UNU-WIDER project on Inequality and Poverty in China.

UNU-WIDER gratefully acknowledges the financial contributions to its research programme by the governments of Denmark (Royal Ministry of Foreign Affairs), Finland (Ministry for Foreign Affairs), Norway (Royal Ministry of Foreign Affairs), Sweden (Swedish International Development Cooperation Agency_Sida) and the United Kingdom (Department for International Development). 


\section{Acronyms}

CCP Chinese Communist Party

CHNS China's Household Nutrition Survey

SOEs state-owned enterprises

SCEs small collective enterprises

LCDs large collective enterprises

OLS ordinary least squared

PRI private sector enterprises

RRR relative risk ratio

The World Institute for Development Economics Research (WIDER) was established by the United Nations University (UNU) as its first research and training centre and started work in Helsinki, Finland in 1985. The Institute undertakes applied research and policy analysis on structural changes affecting the developing and transitional economies, provides a forum for the advocacy of policies leading to robust, equitable and environmentally sustainable growth, and promotes capacity strengthening and training in the field of economic and social policy making. Work is carried out by staff researchers and visiting scholars in Helsinki and through networks of collaborating scholars and institutions around the world.

www.wider.unu.edu

publications@wider.unu.edu

UNU World Institute for Development Economics Research (UNU-WIDER)

Katajanokanlaituri 6 B, 00160 Helsinki, Finland

Camera-ready typescript prepared by Liisa Roponen at UNU-WIDER

The views expressed in this publication are those of the author(s). Publication does not imply endorsement by the Institute or the United Nations University, nor by the programme/project sponsors, of any of the views expressed. 
This paper examines the wage determinants of state-owned, collective-owned, and private sectors in China from 1989 to 1997. Specifically, we estimate the returns to education in different ownership sectors. Education, as a major form of human capital, plays an essential role in enhancing labour's productivity and therefore increasing income, as proclaimed by human capital theory (e.g., Schultz 1961; Becker 1993). Empirically, returns to education are extensively studied around the world, and return rates are generally high (see, for example, Psacharopoulos 1994; Card 1999; Duflo 2001). 1 The literature on China's returns to education is also growing rapidly, and due to dualism, the return rates in rural and urban areas are usually estimated separately. The basic findings on returns to education in the urban areas indicate that these rates were lower than those of other countries in the 1980s and early 1990s and have been increasing ever since. Zhang et al. (2005), for example, estimate the returns to schooling in urban China from 1988 to 2001, and find a robust trend of increasing returns to education. ${ }^{2}$ There are also increasing trends in the rural areas (Zhang, Huang and Rozelle 2002, for example). Broadly speaking, however, the results for rural China are more diverse than those of urban area.3,4 This article is a part of the growing literature seeking to assess the evolution of returns to education on the Chinese labour market. It contributes to the existing literature by estimating the return rates of different ownerships.

In the 1990s, China experienced far-reaching transition, and the coexistence of different ownerships was one prominent feature of this process. The role of private economy as an important complementary part of the whole economy was established by the 14th CCP congress, and subsequently private sectors enlarged vibrantly. Because of China's institutional divergence and labour market segmentation, several questions need to be answered: Do public and private sectors have the same level of human capital? Is human capital equally compensated? To what extents are these caused by labour's selfselection, given that the returns are different?

Articles closely related to these questions include Zhao (2002) and Dong and Bowles (2002). Zhao's (2002) results indicate that in 1996, returns to education in foreign

1 In Psacharopoulos' (1994) survey, the world average return to education is about 10 per cent and 9.6 per cent in Asia. A more recent research (Duflo 2001) on Indonesia finds that the return to education is about 6 10 per cent, after coping with endogeneity problem. As for health, Duncan and Strauss's (1997) research on Brazil shows a significant effect of health and nutrition on wages. For a more recent survey on the return to education in developed countries, see Card (1999).

2 Related literature includes Appleton, Song and Xia (2005); Maurer-Fazio (1999); Johnson and Chow (1997) and Liu (1998).

3 de Brauw et al. (2002) provide a comprehensive survey on rural return to education.

4 See, for example, Meng (1996, 2000); Zhang, Huang and Rozelle (2002); Parish, Zhe and Li (1995) and Wei (2004). Meng (1996, 2000) finds that non-market factors, instead of human capital, play major roles in wage determination. Research by Zhang, Huang and Rozelle (2002) indicates that during the period between late 1980 to mid 1990s, return to education increase, from insignificant rates in 1988 to 6 10 per cent in 1996. Wei (2004) analyses the effects of both education and health, and finds that the return to education is insignificant in 1993, even after addressing the selection problem. As for rural regions, de Brauw et al.'s survey indicates that the return to education is about $0 \sim 6$ per cent. 
invested enterprises are higher than those of state-owned enterprises (SOEs), urban collective enterprises and domestic private enterprises. Dong and Bowles (2002), using survey data from Dalian and Xiamen, analyse the wage-setting behaviour of four types of enterprises: (i) state-owned enterprises, (ii) township and village enterprises, (iii) joint ventures, and (iv) foreign-invested firms in China's light consumer goods industry in 1998. They find no significant difference in the returns to education among the four types of firms. Taking the potential non-random problem into consideration, both articles use Heckman's two-step method to correct selection bias. 5

This paper contributes to this branch of literature by examining another dataset, which is more representative of China's labour market and covers a longer period. Using China's Household Nutrition Survey (CHNS), we find that the returns to education are around 0-2 per cent in the early 1990s in all sectors, for both rural and urban regions. The case is different in 1997, and the returns to education range between 4.5-4.8 per cent in the private sectors while those of other sectors remain low. These results indicate that different sectors have different compensation mechanisms and that the labour market is segmented. We also correct for possible selection bias with a two-step switching model, 6 which can be used to estimate wage equations for more than two categories simultaneously.

In the next section, using the CHNS, we show the patterns of human capital and wage distribution in the different sectors. The econometric results of OLS are presented in section 3; selection bias and the rural-urban divide are addressed in section 4 . Section 5 , using the multinomial logit regression, analyses the role of human capital in sector choice. Section 6 concludes.

\section{Data and model specification}

\subsection{Data description}

The data come from CHNS, conducted by an international team of experts on nutrition, public health, economics, sociology, Chinese studies, and demography. The survey covers about 16,000 individuals from more than 3,000 households in nine provinces including Liaoning, Heilongjiang, Jiangsu, Shandong, Henan, Hubei, Hunan, Guangxi, and Guizhou.7 These provinces vary greatly in terms of geography and economic performance, and thus CHNS can be regarded as representative of China. This paper uses four waves of this dataset, namely 1989, 1991, 1993 and 1997.

5 Other papers (Chen, Demurger and Fournier 2005; Zhao 2001) also investigate wage determination or wage structure of different ownerships.

6 Switching model is increasingly used by researchers. With this model Lee (1978) analyses wage mechanisms between union and non-union sectors; Trost (1977) analyses the difference in expenditure behavior of home owners and renters. Robinson and Tomes (1984) examine the wage differential between public and non-public sectors. With regard to multi-choice models, Hay (1980) analyses the occupation choice decision, Dong and Bowels (2002) review the wage mechanisms for different kinds of firms, but due paucity of data, they confine their research to two types of firms in using Heckman's two stage model.

7 In 1997, Heilongjiang replaced Liaoning. 
Because we focus on wage determination, only individuals with wage income are included. The remaining sample is then divided into four subsets according to the survey's ownership categories: state-owned enterprises (SOEs), small collective enterprises (SCEs), large collective enterprises (LCEs), and the private sector (PRI). 8 Table 1 reports the labour shares of different ownerships from 1989 to 1997. In the rural regions, private sectors account for a considerable fraction of the labourforce, and this increases slightly from 23 per cent in 1989 to 26 per cent in 1997. In urban areas, public sectors dominate from 1989 to 1997, with the state-owned sector taking 60-70 per cent of the labour force. The share of private sector increases, however, from 5 per cent in 1989 to 12 per cent in 1997.9

Table 2 reports summary statistics. As for years of schooling (also depicted in Figure 1), several points need to be mentioned. First, the education level is the highest in SOEs from 1989 to 1997. Taking the 1991 as an example, the descending sequence is SOEs, LCEs, SCEs, and PRI in the rural regions, with the average education levels being 9.0, 7.5, 7.3, and 7.1 years. The case of the urban areas is different, with the SCEs and PRI reversing their position after 1991. Second, except for the SCE sectors, education levels in all sectors are higher in the urban areas than in the rural. Third, education levels increase in all sectors during this period. Another form of human capital is health, for which height is an indicator (e.g., Duncan and Strauss 1997). Height is invariably higher in the SOEs than in other sectors (depicted in Figure 2). Table 2 also reports the hours worked per day. Daily working hours in the SOEs tend to be less than those in other ownership firms.

Table 1

Labour shares of different ownerships

\begin{tabular}{|c|c|c|c|c|c|c|c|c|}
\hline \multirow[b]{2}{*}{ Type of work unit } & \multicolumn{2}{|c|}{1989} & \multicolumn{2}{|c|}{1991} & \multicolumn{2}{|c|}{1993} & \multicolumn{2}{|c|}{1997} \\
\hline & Persons & $\%$ & Persons & $\%$ & Persons & $\%$ & Persons & $\%$ \\
\hline & \multicolumn{8}{|c|}{ Rural } \\
\hline State owned (SOEs) & 478 & 35.83 & 504 & 35.07 & 450 & 33.28 & 586 & 40.89 \\
\hline Small collective (SCEs) & 404 & 30.28 & 396 & 27.56 & 377 & 27.88 & 311 & 21.7 \\
\hline Large collective (LCES) & 145 & 10.87 & 184 & 12.8 & 211 & 15.61 & 164 & 11.44 \\
\hline Private (PRI) & 307 & 23.01 & 353 & 24.57 & 314 & 23.22 & 372 & 25.96 \\
\hline \multirow[t]{2}{*}{ Total } & 1,334 & 100.00 & 1,437 & 100.00 & 1,352 & 100.00 & 1,433 & 100.00 \\
\hline & \multicolumn{8}{|c|}{ Urban } \\
\hline State owned (SOEs) & 1,294 & 64.22 & 1,185 & 66.05 & 919 & 65.83 & 809 & 60.87 \\
\hline Small collective (SCEs) & 246 & 12.21 & 212 & 11.82 & 143 & 10.24 & 169 & 12.72 \\
\hline Large collective (LCES) & 373 & 18.51 & 320 & 17.84 & 255 & 18.27 & 195 & 14.67 \\
\hline Private (PRI) & 102 & 5.06 & 77 & 4.29 & 79 & 5.66 & 156 & 11.74 \\
\hline Total & 2,015 & 100.00 & 1,794 & 100.00 & 1,396 & 100.00 & 1,329 & 100.00 \\
\hline
\end{tabular}

Source: Compiled by the author, based on data from CHNS.

8 The classification of SOEs, SCEs and LCEs is consistent in the survey from 1989 to 1997. While 'private' is the only category remaining in 1989, it is further subdivided into 'private or individual' and 'joint venture' or 'three capital'. For consistency, this paper still treats these subgroups as private sector.

9 If Heilongjian had not replaced Liaoning in 1997, the labour share in the private sector would have been higher than reported in Table 1, because the SOEs have a greater labour share in Heilongjiang. In 1996 in Heilongjiang and Liaoning, the labour shares in SOEs are 39 per cent and 33 per cent, respectively (NBS 1997: Tables 4-3 and 4-10). 
Although public sectors, especially SOEs, are at an advantage in terms of human capital level, their wage levels are low compared to the private sectors. Take the rural sample for 1991, for example, hourly wages are 0.5, 0.6, 0.5, and 1.1 yuan in the SOEs, SCEs, LCEs, and PRI. With the exception of 1989, the standard errors of hourly wages are larger for the private sector than for other ownerships. Table 3 also calculates the 10 per cent, 25 per cent, 50 per cent, 75 per cent, and 90 per cent percentiles of the wages for various ownerships. It is obvious that wages in the private sectors show greater dispersion than in other sectors. In the rural areas, the wage level in 1991 is around 0.3 yuan/hour at the 10 per cent percentile level in all sectors; at the 90 per cent percentile level, however, wage level in the private sectors is about 1.9 yuan/hour, i.e., 2.5, 1.9, and 2.5 times that of SOEs, SCEs, and LCEs, respectively. The patterns in the urban area and for other survey years are similar.

Table 2

Summary statistics

\begin{tabular}{|c|c|c|c|c|c|c|c|c|c|}
\hline & & \multicolumn{2}{|c|}{ SOES } & \multicolumn{2}{|c|}{ SCES } & \multicolumn{2}{|c|}{ LCEs } & \multicolumn{2}{|c|}{ PRI } \\
\hline & & Mean & s.e. & Mean & s.e. & Mean & s.e. & Mean & s.e. \\
\hline \multirow[t]{5}{*}{1989} & Hourly wage (yuan) & 1.1 & 2.3 & 0.7 & 1.4 & 0.6 & 1.1 & 1.1 & 1.6 \\
\hline & Working hours per day & 7.9 & 0.8 & 8.0 & 1.5 & 8.1 & 0.9 & 7.6 & 2.6 \\
\hline & Schooling (yrs) & 8.7 & 3.8 & 7.3 & 3.5 & 7.5 & 3.1 & 6.5 & 3.1 \\
\hline & Height (cm) & 165.1 & 7.8 & 163.2 & 7.4 & 163.8 & 7.6 & 163.3 & 6.8 \\
\hline & Age & 35.8 & 11.9 & 31.9 & 11.0 & 32.3 & 10.6 & 34.1 & 12.0 \\
\hline \multirow[t]{5}{*}{1991} & Hourly wage (yuan) & 0.5 & 0.3 & 0.6 & 0.4 & 0.5 & 0.2 & 1.1 & 2.0 \\
\hline & Working hours per day & 8.0 & 0.8 & 8.2 & 1.1 & 8.1 & 0.8 & 8.0 & 2.5 \\
\hline & Schooling (yrs) & 9.0 & 3.5 & 7.3 & 3.3 & 7.5 & 2.9 & 7.1 & 3.1 \\
\hline & Height $(\mathrm{cm})$ & 163.6 & 10.8 & 162.3 & 7.2 & 162.8 & 10.5 & 161.7 & 11.3 \\
\hline & Age & 34.1 & 11.0 & 33.3 & 11.2 & 32.9 & 10.7 & 32.1 & 11.6 \\
\hline \multirow[t]{5}{*}{1993} & Hourly wage (yuan) & 0.7 & 0.4 & 0.8 & 0.4 & 0.8 & 0.5 & 1.4 & 1.8 \\
\hline & Working hours per day & 7.9 & 0.9 & 8.1 & 2.9 & 8.6 & 5.2 & 8.2 & 3.9 \\
\hline & Schooling (yrs) & 9.1 & 3.6 & 7.6 & 3.1 & 7.7 & 2.6 & 7.0 & 2.8 \\
\hline & Height $(\mathrm{cm})$ & 164.5 & 8.1 & 162.8 & 7.6 & 161.6 & 7.4 & 162.3 & 7.3 \\
\hline & Age & 35.6 & 11.2 & 34.3 & 11.2 & 32.1 & 11.4 & 33.3 & 11.4 \\
\hline \multirow[t]{5}{*}{1997} & Hourly wage (yuan) & 1.0 & 0.6 & 1.1 & 1.1 & 1.2 & 1.2 & 1.4 & 1.4 \\
\hline & Working hours per day & 7.7 & 1.0 & 7.9 & 1.5 & 8.0 & 1.7 & 8.5 & 1.8 \\
\hline & Schooling (yrs) & 10.3 & 2.5 & 8.2 & 2.7 & 9.1 & 2.8 & 8.0 & 3.0 \\
\hline & Age & 36.2 & 10.4 & 39.3 & 10.5 & 35.8 & 10.5 & 35.9 & 13.0 \\
\hline & & \multicolumn{8}{|c|}{ Urban } \\
\hline \multirow[t]{5}{*}{1989} & Hourly wage (yuan) & 0.6 & 1.3 & 0.6 & 1.3 & 0.7 & 1.8 & 0.7 & 0.7 \\
\hline & Working hours per day & 7.9 & 1.0 & 8.0 & 1.5 & 8.0 & 1.2 & 8.3 & 2.2 \\
\hline & Schooling (yrs) & 9.5 & 3.6 & 6.9 & 3.5 & 8.0 & 3.2 & 6.7 & 3.4 \\
\hline & Height $(\mathrm{cm})$ & 162.9 & 8.0 & 162.2 & 8.4 & 162.0 & 7.6 & 160.4 & 7.4 \\
\hline & Age & 36.1 & 12.1 & 35.5 & 12.5 & 35.2 & 12.4 & 35.5 & 15.1 \\
\hline \multirow[t]{5}{*}{1991} & Hourly wage (yuan) & 0.5 & 0.2 & 0.5 & 0.3 & 0.4 & 0.3 & 0.8 & 0.5 \\
\hline & Working hours per day & 7.9 & 0.7 & 8.2 & 1.2 & 7.9 & 0.9 & 8.2 & 1.9 \\
\hline & Schooling (yrs) & 9.9 & 3.2 & 7.1 & 3.2 & 8.3 & 3.0 & 7.5 & 3.4 \\
\hline & Height $(\mathrm{cm})$ & 163.5 & 8.7 & 162.1 & 7.5 & 161.6 & 12.0 & 161.0 & 7.5 \\
\hline & Age & 35.8 & 11.2 & 36.5 & 11.7 & 33.4 & 11.3 & 37.1 & 12.8 \\
\hline \multirow[t]{5}{*}{1993} & Hourly wage (yuan) & 0.7 & 0.8 & 0.7 & 0.5 & 0.7 & 2.1 & 2.7 & 10.8 \\
\hline & Working hours per day & 7.9 & 2.0 & 8.4 & 2.2 & 7.7 & 1.1 & 8.1 & 2.1 \\
\hline & Schooling (yrs) & 9.9 & 3.3 & 7.2 & 3.1 & 8.5 & 2.8 & 7.9 & 3.3 \\
\hline & Height (cm) & 163.4 & 9.6 & 162.2 & 14.6 & 163.0 & 8.1 & 162.0 & 7.7 \\
\hline & Age & 37.5 & 10.9 & 37.5 & 10.4 & 34.4 & 10.6 & 35.6 & 12.1 \\
\hline \multirow[t]{4}{*}{1997} & Hourly wage (yuan) & 1.1 & 0.6 & 1.3 & 1.6 & 1.0 & 0.4 & 1.5 & 1.5 \\
\hline & Working hours per day & 7.8 & 0.9 & 7.7 & 1.8 & 8.0 & 0.8 & 8.7 & 2.0 \\
\hline & Schooling (yrs) & 10.9 & 3.0 & 8.2 & 3.3 & 9.7 & 2.8 & 9.0 & 3.1 \\
\hline & Age & 37.5 & 10.3 & 38.8 & 11.4 & 36.4 & 9.7 & 34.2 & 11.0 \\
\hline
\end{tabular}

Source: Compiled by the author, based on data from CHNS. 
Table 3

Wage distribution (unit: yuan)

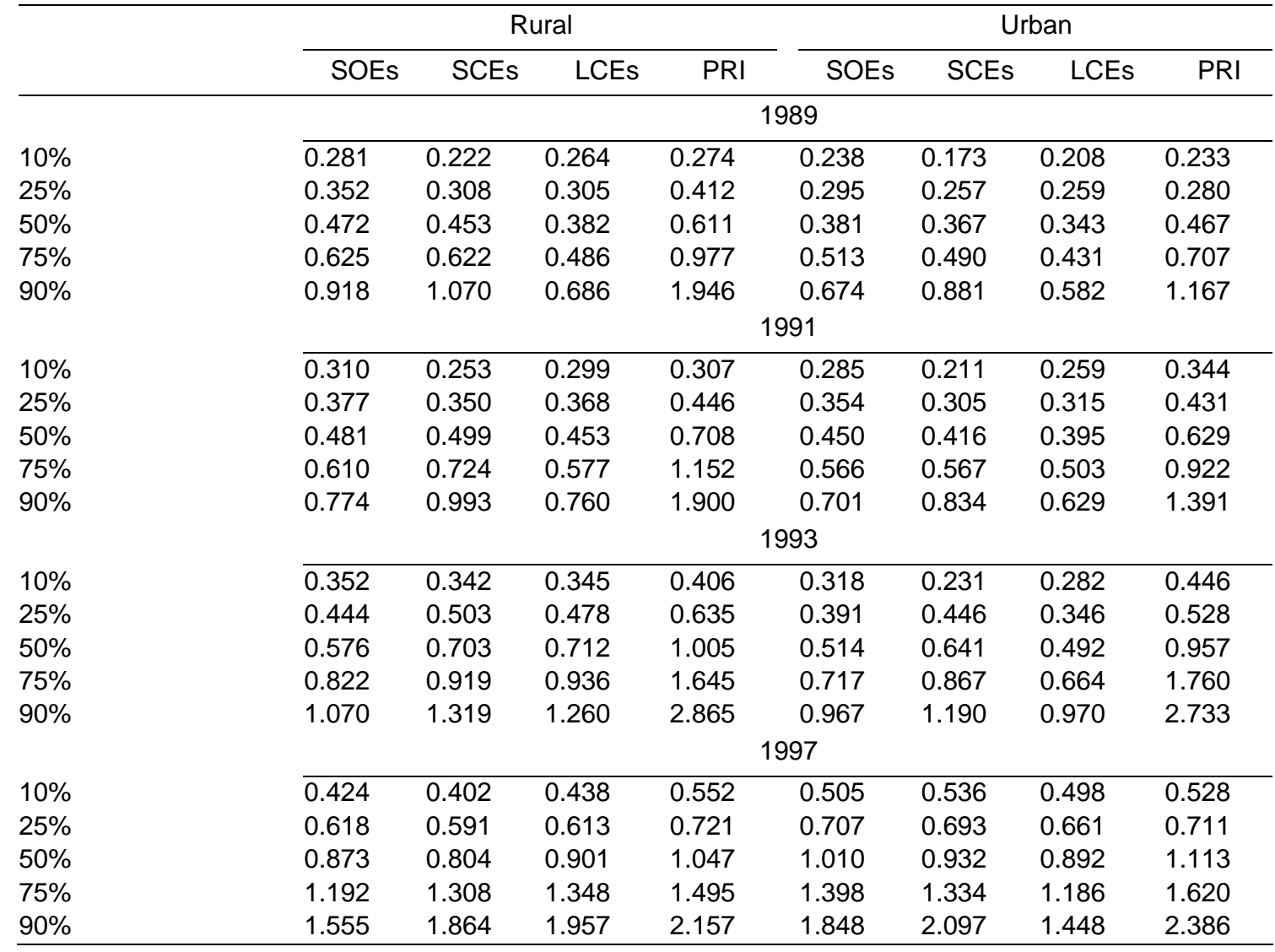

Source: Compiled by the author, based on data from CHNS.

Figure 1

Years of schooling from 1989 to 1997
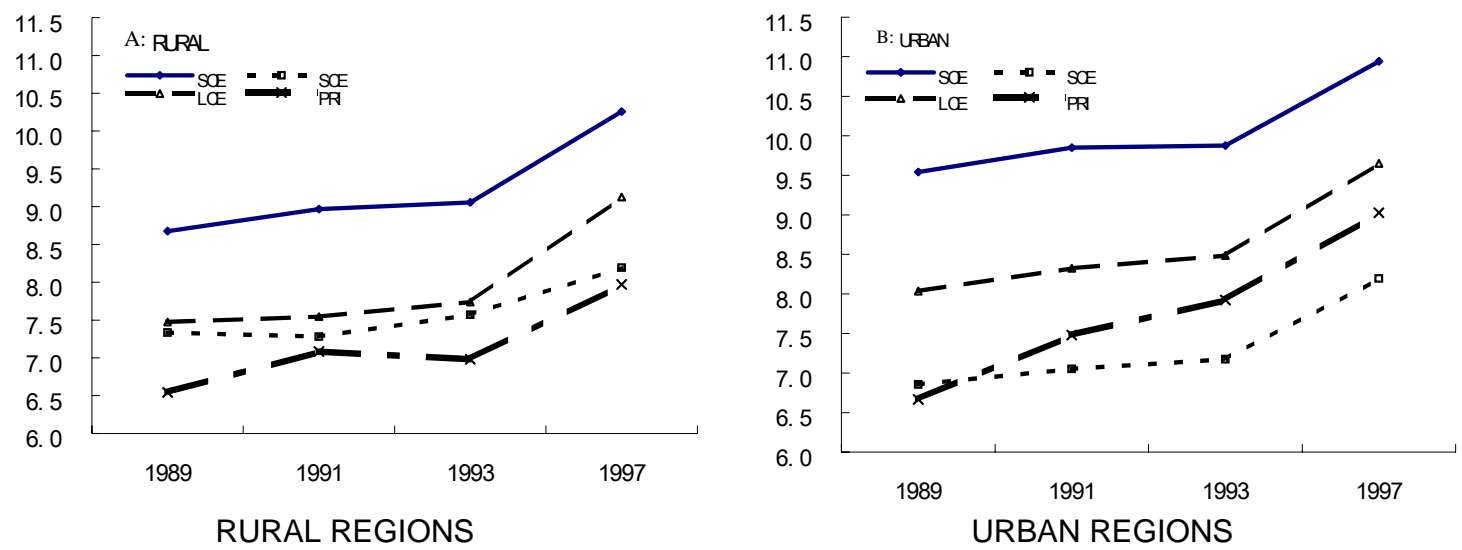

Source: Computed by the author, based on data from CHNS.

The patterns of wage distribution may be partly related to the definition of wages, as bonuses and subsidies are excluded in the questionnaire. This makes it hard to compare the results of this paper to others. 10 However, because wages constitute the most important part of one's income, and bonuses and subsidies may be subject to factors

10 For example, Zhang et al. (2005), and the literature mentioned therein. 
such as wage regulations, employer's profitability, and economic fluctuations, our discussion in this paper is confined to pure wages.

Figure 2

Height of Labourers in different ownerships (Unit: $\mathrm{cm}$ )
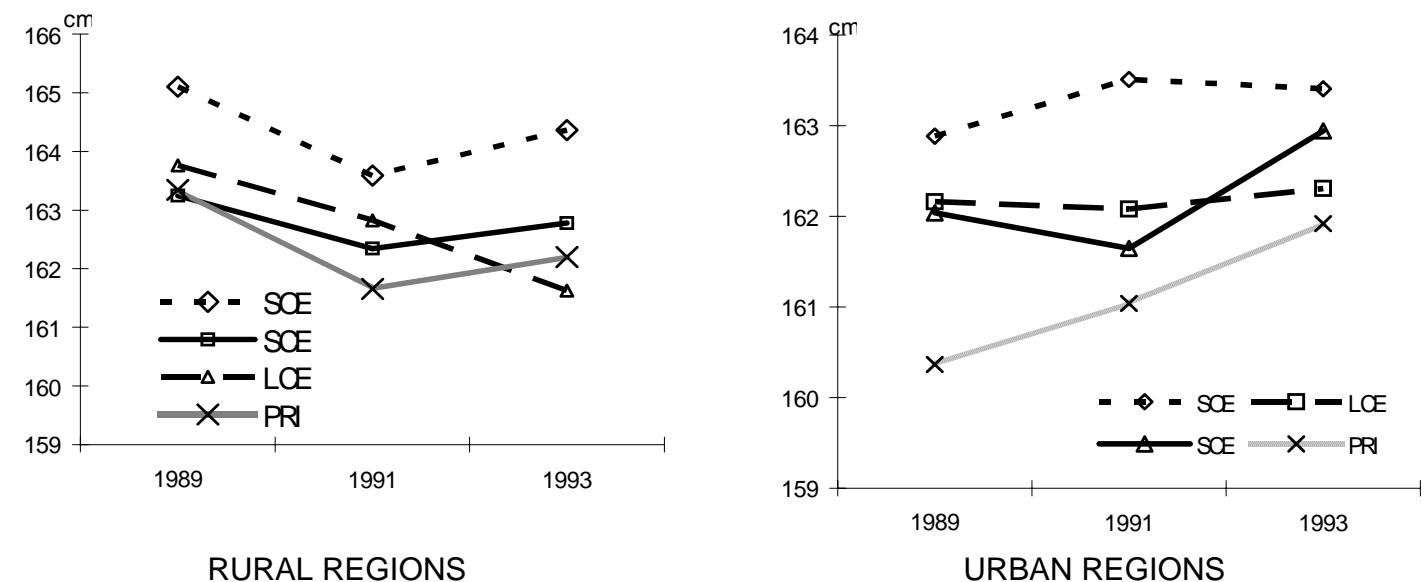

RURAL REGIONS

Source: Computed by the author, based on data from CHNS.

\subsection{Model specification}

The model we estimate is an extended Mincer (1974) equation:

$$
\ln \left(\text { wage }_{s i}\right)=H_{s i} \beta_{s 1}+X_{s i} \beta_{s 2}+u_{s i}
$$

Where $s$ refers to different sectors with $s=1,2,3,4$ representing SOEs, SCEs, LCEs, and PRI, respectively; $H$ is human capital vector, which may include education, health, experience and experience square. We use the years of formal schooling and height as proxies of education and health, respectively. 11 Experience is calculated according to Mincer (1974): experience=age - (years of schooling) -6. In addition, $X$ controls for such non-market factors such as gender, province dummies, occupation and constant; $E\left(u_{s} \mid H_{s}, X_{s}\right)=0, \ln (\text { wage })_{s i}$ is the log form of observation i's wage, which can be observed only when individual $i$ is in the sector $s$ (selection bias is discussed later). We estimate wage equations separately for each sector and year to allow the returns to education to differ. One alternative method is to include interaction terms in one setting instead of estimating them separately. Although using only one equation seems to be more efficient than separate estimations, both are essentially the same. in order to be consistent with the switching model in section 4 , we estimate the wage equation by sectors.

In the remaining sector, we estimate the wage equations by year rather than pooling different years (so that individual fixed effects can be controlled for). On the one hand, the sample changes considerably, especially in 1997, when Liaoning was replaced by Heilongjiang province. On the other hand, as education itself is time invariant, we can at most estimate the return differentials between various years by interacting education with year dummies. Furthermore, fixed effects model makes it difficult to correct for selectivity bias.

11 Height is widely used as a proxy for health, see, for example, Duncan and Strauss (1997); Strauss and Duncan (1998), and Fogel (1994). 


\section{OLS results}

\subsection{Pooling results}

First we assume that wage determination is identical in all sectors, and this allows us to run a pooling regression. We just control the ownership dummies to see their effects on wages. The results are presented in Table 4. When the ownership dummies are not controlled, the adjusted r-squares are around 13.4 per cent, 24.5 per cent, 16.5 per cent and 15.8 per cent in 1989, 1991, 1993, and 1997, respectively. The adjusted r-squares increased by 2.2, 1.7, 6.1, and 1.6 percentage points when they are included. The results also indicate that differences in the wage levels exist mainly between the private and

Table 4

Wage equation (pooling results)

Dependent variable $=\log$ (hour wage)

\begin{tabular}{|c|c|c|c|c|c|c|c|c|}
\hline & \multicolumn{2}{|c|}{1989} & \multicolumn{2}{|c|}{1991} & \multicolumn{2}{|c|}{1993} & \multicolumn{2}{|c|}{1997} \\
\hline & 1 & 2 & 3 & 4 & 5 & 6 & 7 & 8 \\
\hline \multirow[t]{2}{*}{ Schooling } & 0.011 & $0.014^{\star \star}$ & $0.015^{\star \star \star}$ & $0.016^{\star \star \star}$ & $0.016^{\star \star \star}$ & $0.023^{\star \star \star}$ & $0.013^{\star \star}$ & $0.013^{\star \star}$ \\
\hline & $(0.007)$ & $(0.007)$ & $(0.003)$ & $(0.003)$ & $(0.005)$ & $(0.005)$ & $(0.005)$ & $(0.005)$ \\
\hline \multirow[t]{2}{*}{ Experience } & $0.022^{\star \star *}$ & $0.023^{\star \star \star}$ & $0.026^{\star \star \star}$ & $0.026^{\star \star \star}$ & $0.028^{\star \star \star}$ & $0.028^{\star \star *}$ & $0.027^{\star \star \star}$ & $0.030 * * *$ \\
\hline & $(0.008)$ & $(0.008)$ & $(0.002)$ & $(0.002)$ & $(0.003)$ & $(0.003)$ & $(0.004)$ & $(0.004)$ \\
\hline \multirow[t]{2}{*}{ Experience square/100 } & -0.015 & -0.015 & $-0.030^{* * *}$ & $-0.031^{\star \star *}$ & $-0.037^{\star \star \star}$ & $-0.035^{\star \star \star}$ & $-0.048^{\star * \star}$ & $-0.052^{\star \star *}$ \\
\hline & $(0.021)$ & $(0.020)$ & $(0.004)$ & $(0.004)$ & $(0.006)$ & $(0.006)$ & $(0.007)$ & $(0.007)$ \\
\hline \multirow[t]{2}{*}{ Ln(height) } & 0.135 & 0.261 & 0.244 & 0.277 & $0.666^{\star *}$ & $0.622^{\star \star}$ & & \\
\hline & $(0.214)$ & $(0.212)$ & $(0.205)$ & $(0.203)$ & $(0.284)$ & $(0.273)$ & & \\
\hline Female & $-0.095^{\star \star *}$ & $-0.085^{\star \star}$ & $-0.120^{\star \star \star}$ & $-0.121^{* \star *}$ & $-0.092^{\star \star \star}$ & $-0.081^{\star \star \star}$ & $-0.155^{\star \star \star}$ & $-0.155^{\star \star \star}$ \\
\hline \multicolumn{9}{|c|}{$\begin{array}{l}\text { Liaoning (' } 89, \text { '91, '93) } \\
\text { or Heilongjiang ('97) omitted }\end{array}$} \\
\hline & $(0.036)$ & $(0.035)$ & $(0.022)$ & $(0.022)$ & $(0.031)$ & $(0.030)$ & $(0.023)$ & $(0.023)$ \\
\hline \multirow[t]{2}{*}{ Jiangsu } & $-0.103^{*}$ & $-0.141^{\star \star \star}$ & $-0.059^{\star}$ & $-0.077^{\star \star}$ & $0.120^{\star \star \star}$ & 0.047 & $0.310^{\star \star *}$ & $0.320^{\star \star \star}$ \\
\hline & $(0.054)$ & $(0.055)$ & $(0.030)$ & $(0.031)$ & $(0.043)$ & $(0.043)$ & $(0.049)$ & $(0.049)$ \\
\hline \multirow[t]{2}{*}{ Shandong } & $0.157^{\star \star}$ & $0.127^{*}$ & 0.000 & -0.011 & $0.126^{\star \star \star}$ & $0.115^{\star \star}$ & $0.141^{\star \star}$ & $0.167^{\star \star \star}$ \\
\hline & $(0.064)$ & $(0.067)$ & $(0.032)$ & $(0.032)$ & $(0.048)$ & $(0.047)$ & $(0.055)$ & $(0.056)$ \\
\hline \multirow[t]{2}{*}{ Henan } & $0.139^{\star \star}$ & 0.082 & $0.065^{\star}$ & 0.055 & -0.026 & -0.067 & $0.186^{\star \star *}$ & $0.194^{\star \star \star}$ \\
\hline & $(0.064)$ & $(0.064)$ & (0.039) & $(0.039)$ & $(0.054)$ & $(0.052)$ & $(0.056)$ & $(0.056)$ \\
\hline \multirow[t]{2}{*}{ Hubei } & 0.053 & 0.021 & $0.126^{\star \star \star}$ & $0.116^{\star \star \star}$ & $0.087^{\star}$ & $0.077^{*}$ & $0.196^{\star \star \star}$ & $0.213^{\star \star \star *}$ \\
\hline & (0.058) & $(0.058)$ & $(0.033)$ & $(0.033)$ & $(0.046)$ & $(0.045)$ & $(0.052)$ & $(0.052)$ \\
\hline \multirow[t]{2}{*}{ Hunan } & $0.273^{\star \star \star}$ & $0.219^{\star \star \star}$ & $0.123^{\star \star \star}$ & $0.097^{\star \star *}$ & $0.282^{\star \star \star}$ & $0.241^{\star \star \star}$ & $0.458^{\star \star \star}$ & $0.442^{\star * *}$ \\
\hline & $(0.059)$ & $(0.059)$ & $(0.033)$ & $(0.032)$ & $(0.047)$ & $(0.045)$ & $(0.054)$ & $(0.054)$ \\
\hline \multirow[t]{2}{*}{ Guangxi } & -0.013 & -0.051 & -0.056 & $-0.080^{\star \star}$ & $0.137^{\star \star \star}$ & 0.071 & $0.108^{\star \star}$ & $0.093^{*}$ \\
\hline & (0.072) & $(0.071)$ & $(0.036)$ & $(0.036)$ & $(0.049)$ & $(0.047)$ & $(0.053)$ & $(0.052)$ \\
\hline \multirow[t]{2}{*}{ Guizhou } & 0.094 & 0.055 & $-0.071^{*}$ & $-0.073^{*}$ & $0.094^{\star}$ & 0.045 & $0.098^{*}$ & $0.101^{*}$ \\
\hline & $(0.066)$ & $(0.066)$ & $(0.038)$ & $(0.038)$ & $(0.052)$ & $(0.051)$ & $(0.057)$ & $(0.057)$ \\
\hline \multicolumn{9}{|c|}{ State-owned sector omitted } \\
\hline \multirow[t]{2}{*}{ Small collective } & & 0.075 & & $0.050 * \star$ & & $0.122^{* * *}$ & & -0.031 \\
\hline & & $(0.047)$ & & $(0.025)$ & & $(0.035)$ & & $(0.041)$ \\
\hline \multirow[t]{2}{*}{ Large collective } & & -0.003 & & 0.003 & & $0.073^{\star \star}$ & & -0.025 \\
\hline & & $(0.046)$ & & $(0.025)$ & & $(0.034)$ & & $(0.036)$ \\
\hline \multirow[t]{2}{*}{ Private sector } & & $0.519 * \star \star$ & & $0.359^{\star \star \star}$ & & $0.657^{\star \star \star}$ & & $0.207^{\star \star \star}$ \\
\hline & & $(0.082)$ & & $(0.046)$ & & $(0.049)$ & & $(0.044)$ \\
\hline R2_a & 0.134 & 0.156 & 0.245 & 0.262 & 0.165 & 0.226 & 0.154 & 0.168 \\
\hline $\mathrm{N}$ & 1506 & 1506 & 2688 & 2688 & 2302 & 2302 & 2233 & 2233 \\
\hline
\end{tabular}

Note: Occupation, urban dummy are controlled. *significant at $10 \%$ level, ${ }^{\star \star}$ significant $5 \%$ level, ${ }^{\star * *}$ significant at $1 \%$ level. Standard errors in parenthesis, constant not reported. 
Table 5

Wage equations of different sectors

Dependent variable=log (hourly wage)

\begin{tabular}{|c|c|c|c|c|c|c|c|c|c|c|c|c|}
\hline & \multicolumn{4}{|c|}{1991} & \multicolumn{4}{|c|}{1993} & \multicolumn{4}{|c|}{1997} \\
\hline & SOES & SCES & LCES & PRI & SOES & SCES & LCES & PRI & SOES & SCES & LCES & PRI \\
\hline & \multicolumn{12}{|c|}{ PANEL A: OLS } \\
\hline Schooling & $\begin{array}{l}0.020^{* \star *} \\
(0.003)\end{array}$ & $\begin{array}{c}0.006 \\
(0.010)\end{array}$ & $\begin{array}{c}0.012 \\
(0.008)\end{array}$ & $\begin{array}{c}0.011 \\
(0.017)\end{array}$ & $\begin{array}{l}0.023^{\star * *} \\
(0.005)\end{array}$ & $\begin{array}{c}0.014 \\
(0.012)\end{array}$ & $\begin{array}{c}0.005 \\
(0.013)\end{array}$ & $\begin{array}{c}0.024 \\
(0.021)\end{array}$ & $\begin{array}{c}0.006 \\
(0.006)\end{array}$ & $\begin{array}{c}0.008 \\
(0.015)\end{array}$ & $\begin{array}{c}0.004 \\
(0.015)\end{array}$ & $\begin{array}{l}0.041^{\star \star \star} \\
(0.013)\end{array}$ \\
\hline Experience & $\begin{array}{l}0.026^{\star \star \star} \\
(0.002)\end{array}$ & $\begin{array}{l}0.018^{\star \star \star} \\
(0.006)\end{array}$ & $\begin{array}{l}0.017^{\star \star \star} \\
(0.005)\end{array}$ & $\begin{array}{l}0.035^{\star \star \star} \\
(0.012)\end{array}$ & $\begin{array}{l}0.029^{\star \star \star} \\
(0.004)\end{array}$ & $\begin{array}{l}0.028^{\star \star \star} \\
(0.007)\end{array}$ & $\begin{array}{c}0.012 \\
(0.008)\end{array}$ & $\begin{array}{l}0.046^{\star \star \star} \\
(0.015)\end{array}$ & $\begin{array}{l}0.035^{\star \star \star} \\
(0.004)\end{array}$ & $\begin{array}{l}0.026^{\star \star} \\
(0.011)\end{array}$ & $\begin{array}{l}0.022^{\star \star} \\
(0.010)\end{array}$ & $\begin{array}{l}0.028^{\star \star \star \star} \\
(0.007)\end{array}$ \\
\hline Experience square/100 & $\begin{array}{l}-0.020 \star \star \star \\
(0.004)\end{array}$ & $\begin{array}{l}-0.031^{\star \star \star} \\
(0.011)\end{array}$ & $\begin{array}{l}-0.011 \\
(0.009)\end{array}$ & $\begin{array}{l}-0.063^{\star \star \star} \\
(0.022)\end{array}$ & $\begin{array}{l}-0.031^{\star \star \star} \\
(0.007)\end{array}$ & $\begin{array}{l}-0.050^{\star \star \star} \\
(0.013)\end{array}$ & $\begin{array}{l}-0.011 \\
(0.014)\end{array}$ & $\begin{array}{l}-0.072^{\star \star \star} \\
(0.027)\end{array}$ & $\begin{array}{l}-0.064^{\star \star \star} \\
(0.009)\end{array}$ & $\begin{array}{l}-0.048^{\star \star} \\
(0.020)\end{array}$ & $\begin{array}{l}-0.034 \\
(0.023)\end{array}$ & $\begin{array}{l}-0.045^{\star \star \star} \\
(0.012)\end{array}$ \\
\hline Ln(height) & $\begin{array}{l}-0.039 \\
(0.201)\end{array}$ & $\begin{array}{c}0.663 \\
(0.534)\end{array}$ & $\begin{array}{l}-0.164 \\
(0.384)\end{array}$ & $\begin{array}{c}1.964^{*} \\
(1.032)\end{array}$ & $\begin{array}{l}0.633^{\star *} \\
(0.310)\end{array}$ & $\begin{array}{l}1.210^{\star *} \\
(0.539)\end{array}$ & $\begin{array}{l}-0.319 \\
(0.606)\end{array}$ & $\begin{array}{c}2.447 \\
(1.633)\end{array}$ & & & & \\
\hline R2_a & 0.392 & 0.147 & 0.162 & 0.071 & 0.184 & 0.217 & 0.114 & 0.186 & 0.199 & 0.148 & 0.074 & 0.292 \\
\hline \multirow[t]{2}{*}{ N } & 1437 & 522 & 433 & 296 & 1180 & 431 & 393 & 298 & 1201 & 383 & 299 & 350 \\
\hline & \multicolumn{12}{|c|}{ PANEL B: SWITCHING } \\
\hline Schooling & $\begin{array}{l}0.015^{\star \star \star} \\
(0.004)\end{array}$ & $\begin{array}{c}0.001 \\
(0.011)\end{array}$ & $\begin{array}{l}-0.003 \\
(0.011)\end{array}$ & $\begin{array}{l}-0.009 \\
(0.020)\end{array}$ & $\begin{array}{l}0.017^{\star \star \star} \\
(0.007)\end{array}$ & $\begin{array}{c}0.002 \\
(0.014)\end{array}$ & $\begin{array}{l}-0.021 \\
(0.014)\end{array}$ & $\begin{array}{c}0.000 \\
(0.026)\end{array}$ & $\begin{array}{c}0.010 \\
(0.007)\end{array}$ & $\begin{array}{c}0.006 \\
(0.015)\end{array}$ & $\begin{array}{l}-0.001 \\
(0.016)\end{array}$ & $\begin{array}{l}0.033^{\star *} \\
(0.015)\end{array}$ \\
\hline Experience & $\begin{array}{l}0.025^{\star \star \star} \\
(0.003)\end{array}$ & $\begin{array}{l}0.020^{\star \star \star} \\
(0.007)\end{array}$ & $\begin{array}{l}0.014^{\star \star} \\
(0.006)\end{array}$ & $\begin{array}{l}0.031^{\star *} \\
(0.013)\end{array}$ & $\begin{array}{l}0.027^{\star \star \star} \\
(0.005)\end{array}$ & $\begin{array}{l}0.026^{\star \star \star} \\
(0.009)\end{array}$ & $\begin{array}{c}0.000 \\
(0.009)\end{array}$ & $\begin{array}{l}0.042^{\star \star} \\
(0.017)\end{array}$ & $\begin{array}{l}0.036^{\star \star \star} \\
(0.004)\end{array}$ & $\begin{array}{l}0.032^{\star \star *} \\
(0.011)\end{array}$ & $\begin{array}{l}0.021^{\star \star} \\
(0.010)\end{array}$ & $\begin{array}{l}0.024^{\star \star \star} \\
(0.008)\end{array}$ \\
\hline Experience square/100 & 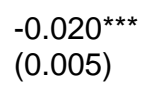 & $\begin{array}{l}-0.037^{\star \star \star} \\
(0.013)\end{array}$ & $\begin{array}{l}-0.011 \\
(0.010)\end{array}$ & $\begin{array}{l}-0.059 \star \star \\
(0.023)\end{array}$ & $\begin{array}{l}-0.032^{\star \star \star} \\
(0.009)\end{array}$ & $\begin{array}{l}-0.049 \star \star \star \\
(0.016)\end{array}$ & $\begin{array}{c}0.001 \\
(0.016)\end{array}$ & $\begin{array}{l}-0.073^{\star \star} \\
(0.031)\end{array}$ & $\begin{array}{l}-0.065^{\star \star \star} \\
(0.009)\end{array}$ & $\begin{array}{l}-0.060^{\star \star \star} \\
(0.021)\end{array}$ & $\begin{array}{l}-0.036 \\
(0.023)\end{array}$ & $\begin{array}{l}-0.039 \star \star \star \star \\
(0.013)\end{array}$ \\
\hline Ln(height) & $\begin{array}{c}0.085 \\
(0.238)\end{array}$ & $\begin{array}{c}1.001^{*} \\
(0.602)\end{array}$ & $\begin{array}{l}-0.277 \\
(0.447)\end{array}$ & $\begin{array}{l}2.523^{\star \star} \\
(1.075)\end{array}$ & $\begin{array}{l}0.655^{\star \star} \\
(0.321)\end{array}$ & $\begin{array}{c}0.992 \\
(0.737)\end{array}$ & $\begin{array}{l}-0.892 \\
(0.593)\end{array}$ & $\begin{array}{c}2.674 \\
(1.773)\end{array}$ & & & & \\
\hline Mills & $\begin{array}{l}-0.053 \\
(0.040)\end{array}$ & $\begin{array}{c}0.060 \\
(0.099)\end{array}$ & $\begin{array}{c}0.131 \\
(0.102)\end{array}$ & $\begin{array}{c}0.251 \\
(0.154)\end{array}$ & $\begin{array}{c}0.001 \\
(0.066)\end{array}$ & $\begin{array}{c}0.250 \\
(0.180)\end{array}$ & $\begin{array}{l}0.340^{\star *} \\
(0.144)\end{array}$ & $\begin{array}{l}0.514^{\star \star \star} \\
(0.193)\end{array}$ & $\begin{array}{c}0.070 \\
(0.061)\end{array}$ & $\begin{array}{c}0.014 \\
(0.083)\end{array}$ & $\begin{array}{c}0.199 \\
(0.139)\end{array}$ & $\begin{array}{c}0.111 \\
(0.089)\end{array}$ \\
\hline R2_a & 0.388 & 0.157 & 0.137 & 0.109 & 0.148 & 0.180 & 0.180 & 0.200 & 0.206 & 0.165 & 0.064 & 0.292 \\
\hline N & 1080 & 463 & 345 & 265 & 953 & 333 & 307 & 249 & 1165 & 359 & 290 & 345 \\
\hline
\end{tabular}

Note: Occupation and province dummies are controlled, *significant at $10 \%$ level, ${ }^{\star *}$ significant $5 \%$ level, ${ }^{* *}$ significant at $1 \%$ level. Standard errors in parenthesis, constant not reported.

Source: Compiled by the author, based on data from CHNS. 
public sectors. Given other conditions, average wage levels of the private sectors are about 51.9 per cent, 35.9 per cent, 65.7 per cent, and 18.3 per cent higher than those of the SOEs. The effect of human capital on wages seems to evolve over time. The returns to education are 1.4 per cent, 1.6 per cent, 2.3 per cent, and 1.8 per cent, respectively, in 1989, 1991, 1993, and 1997, all significant. The return rates are low compared to existing literature on China (Appleton et al. 2005: Zhang et al. 2005). One possible explanation is that our wage data differ from most used the literature, as mentioned in section 2. Health (for which height is a proxy) is also positively related to wages. Labourers who are taller tend to earn more wages, but the effects are significant only in 1993.

Unproductive factors are also important in wage determination. Females earn significantly less than their male counterparts; wage levels also vary significantly between regions, which means that the labour market is geographically segmented. So what has happened in China's labour market? Is it becoming more efficient or not? Using the return to education in this way to evaluate Chinese labour market may be an oversimplification because it applies too strong a restriction in assuming that the returns to education are identical in all sectors. Next we relax this restriction, and estimate the wage equations according to sector and year.

\subsection{Wage determination in different sectors (OLS)}

\section{The results of early $1990 \mathrm{~s}$}

Panel A of Table 5 reports the results of 1991, 1993, and 1997.12 In 1991, the return to education is the highest in SOEs among the four sectors, which is about 2 per cent and significant at the 1 per cent level, whereas in other sectors, it is around 1 per cent and none are significant. Experience, however, seems to be an important factor in determining wage levels in all sectors. But the patterns of wage-experience profiles are different between the SOEs and the private sector. For those with no experience, wage levels increase in the SOEs and the private sectors 2.6 per cent and 3.5 per cent, respectively, with one additional year of experience. Considering the term of experience squared, it takes workers in the private sectors about 30 years to reach the peak wage level. In the SOEs, however, it takes as long as 60 years, which implies that wages in SOEs are continually increasing during the labourer's working life. Further, health plays a more significant role in the private sectors, with the wage elasticity to height being about 2.0 (significant at 10 per cent level). This may be related to the nature of the jobs in the private sector which are more strength demanding.

The returns to education increase from 1991 to 1993 in all sectors except the LCEs, while the return rate in the SOEs increases to 2.3 per cent. Still, experience is an important factor in determining the wage levels in all sectors except LCE, and the patterns of wage-experience profiles are also different between sectors. For those with no experience, wages increase in the SOEs and private sectors 2.9 per cent and 4.6 per cent with one additional year of experience, respectively. Considering the terms of experience squared, it takes workers in the private sectors about 32 years to reach the peak wage level, while it takes as long as 47 years in the SOEs to achieve the peak,

12 The results for 1989 are dropped because the sample size of private sector is rather small. 
which means that there is almost no increase in SOEs wages over the employees' working life. But the pattern is already different for 1991.

The above results seem to indicate an unsatisfactory labour market in the early 1990s. In particular, the role of education in wage determination is not significant, particularly in sectors other than the SOEs. The return rate in the SOEs is extremely low, which may indicate that their wage determination mechanisms are not fully productivity-oriented. Education may merely be a rigid criterion. According to Lardy (1997), the period of the early 1990s coincides with the end of second stage of reforms, which were initiated in the mid-1980s and focused on establishing responsibility between managers and the government. Although some research (Groves et al. 1995) indicates that the managerial labour market improved during this period, the above results tell different story with regard to the whole labour market. But we should be cautious in drawing this conclusion because of the wage data used here.

\section{Wage determination in 1997}

From 1993 onward, China's reform entered a critical stage. In 1992, the main target of economic reform was 'building socialist market economy'. Private economy was recognized as an important complementary part of the whole economy, and henceforth, private economy developed vibrantly. How does affect wage determination of the different ownerships?

The most striking result is that returns to education in private sectors increase to 4.1 per cent (significant at the 1 per cent level), while the return rates in other sectors are only about 1 per cent and insignificant. Experience remains a significant factor in wage determination and the wage-experience profiles are concave, which conform with most of the existing literature. But patterns for the SOEs are different from the results for 1991 and 1993. In 1997, it takes only about 27 years in the SOEs to achieve the peak level of wages.

Thus we can draw a tentative conclusion that the returns to education increase from 1991 to 1997 in the private sectors, while those of other sectors do not. Is the Chinese labour market segmented? Does the gap between public and private sectors reflect different wage mechanisms, which in turn are deeply rooted in the economic regime. Self-selection may be a serious problem if the gap is merely the result of people choosing different sectors.

\section{Self-selection and the rural-urban divide}

\subsection{Sample selection correction of the OLS results}

\section{Model specification of switching regression}

An individual chooses his/her work sector based on their personal attributes, which cannot be controlled for in the wage equation. When these unobservable (uncontrollable) attributes are correlated to education, the estimates may be biased. One possible explanation is that workers with more ability or greater entrepreneurial spirit choose to work in the private sectors rather than the SOEs because of the wage gap (Li 1997). We subsequently use the switching model to correct this bias. The model is as follows (which is heavily borrowed from Maddala 1983). 


$$
\ln \left(\text { wage }_{s i}\right)=H_{s i} \beta_{s 1}+X_{s i} \beta_{s 2}+u_{s i},
$$

Equation (1) is exactly the same as in section 2.

$$
I_{s i}^{*}=z_{s i} \gamma+\eta_{s i}
$$

Equation (2) is a latent decision equation, and $I_{s i}^{*}$ is unobservable. What we can actually observe is $I$, which is a multi-value variable with $I=s$ when an individual works in sector $s$ and we have:

$I=s$ if and only if $I_{s}^{*}>\operatorname{MaxI}_{j}^{*},(j=1,2,3,4$, and $j \neq s)$, further we assume

$\varepsilon_{s}=\operatorname{MaxI}_{j}^{*}-\eta_{s},(j=1,2,3,4$, and $j \neq s)$ so we have $I=s$ if and only if $\varepsilon_{s}<z_{s} \gamma$. If we assume that $\eta_{j}$ have identical independent distribution, and the cumulative distribution function is as $F\left(\eta_{i}<c\right)=\exp [-\exp (-c)]$, we have $\varepsilon_{s}$ 's distribution function :

$$
F_{s}(\varepsilon)=\operatorname{Pr}\left(\varepsilon_{s}<\varepsilon\right)=\frac{\exp (\varepsilon)}{\exp (\varepsilon)+\sum_{j \neq s} \exp \left(z_{j} \gamma\right)} 。
$$

For every wage equation of sector $s, \ln \left(\right.$ wage $\left._{s i}\right)$ can be observed if and only if $\varepsilon_{s}<z_{s} \gamma$. Further assume:

$$
\varepsilon_{s}^{*}=J_{s}\left(\varepsilon_{s}\right)=\Phi^{-1}\left[F_{s}(\varepsilon)\right] 。
$$

So we can use OLS to estimate the following equation to obtain the returns to education of different sectors.

$$
E\left(\operatorname{lwg}_{s} \mid H_{s}, X_{s}, \mathcal{E}_{s}<z_{s} \gamma\right)=H_{s} \beta_{s 1}+X_{s} \beta_{s 2}-\sigma_{s} \rho_{s} \phi\left[J_{s}\left(z_{s} \gamma\right)\right] / F_{s}\left(z_{s} \gamma\right)+v_{s}
$$

where, $\sigma_{s}^{2}=\operatorname{var}\left(u_{s}\right), \rho_{s}$ is the correlation coefficient between $u_{s}$ and $\varepsilon_{s}^{*} \cdot \hat{\gamma}$ and $m=\phi\left[J_{s}\left(z_{s} \hat{\gamma}\right)\right] / F_{s}\left(z_{s} \hat{\gamma}\right)$ can be obtained by first estimating a multinomial logit model. 13

To identify the sector choice behaviour, vector $z$ includes information such as family size, household's per capita income, community's population density and infrastructure, proportions of the labourforce in agriculture and in other employment in 1989, 1991, and 1993. For 1997, however, we only have such information as family size, sex ratio, marital status, urban dummy, and certain family assets. Formally, the selection bias is

13 To be precise, what we should estimate is conditional logit model (see Maddala 1983). Because we have information only at the individual level, we use multinomial logit, which is a special version of the conditional logit model (Wooldridge 2002: 501). 
caused by $\rho_{s}$, the correlation coefficient between $u_{s}$ and $\varepsilon_{s}^{*}$. If $\rho_{s}=0$, the results are just the same as that from (1), but when $\rho_{s} \neq 0$, the results of (1) and (3) are different. Intuitively, when $\rho_{s} \neq 0$, the unobservable part in the sector choice stage is correlated with the unobservable part at the wage determination stage. For example, high-ability labour may choose to work in the private sector, and high-ability workers are also better-educated and have higher wages. If this is the case, the return rate differentials are merely the result of sorting, not the results of labour market segmentation. The essence of switching regression or Heckman's two stage model is using the predicted probability of sector choice to serve as proxy for the unobserved part in the wage equation or we can think of using it to absorb the unobservable part in the wage equation. So the coefficient of $m$ reflects the existence of sample selection.

\section{Results of switching regression}

Compared with OLS results, although the coefficients of switching regressions (panel B of Table 5) change to some extent, the patterns of coefficient differentials between the different sectors do not. The returns to education in the switching models are generally lower than their OLS counterparts. In 1991, the returns to education in SOEs are 1.5 per cent (significant at 1 per cent level), while those of others are around zero and become even negative (all insignificant). In 1993, the returns to education in SOEs decrease from 2.3 per cent in OLS to 1.7 per cent; while those of SCEs and PRI decrease to around zero, and on the part of SCEs even decrease to -2 per cent. Possible explanation is that the added parts in the switching regression represent some attributes that positively correlated with education. These attributes may include such factors as ability, family backgrounds and so on. The switching regression results of 1997 are similar to those of the OLS. The coefficients in the switching models change slightly from their OLS counterparts. One additional year of education in the private sectors significantly increases one's income by 3.3 per cent, and the coefficients for other ownerships are about zero and insignificant.

Experience remains significant in most of the switching regressions. Almost all the wage-experience profiles are inverted $U$ shaped except for LCEs in 1993. As is observed in the OLS regressions, the wage-experience profiles have different shapes. In the early 1990s (1991 and 1993), wages increase faster in private sectors for those with zero year of experience than in public sectors: wages increase 3.1 per cent and 2.5 per cent with one additional year of experience in the private and SOE sectors, respectively in 1991, and 4.2 per cent and 2.1 per cent in 1993. As individual's experience accumulates, the growth of wages slows down because the coefficients of experience squared are negative, and after a certain threshold, wage levels begin to decline. In the early 1990s, it takes almost 40 (results for 1993) to 60 years (results for 1991) to reach a peak level in SOEs, but takes about 25-30 years in the private sectors. The results for the SOEs during this period are at odds with the human capital theory projection, and this suggest that wages in the SOEs are more rigid. In 1997, however, the reverse is true. Wages increase faster in the SOEs than in private sectors for workers with zero experience, moreover, taking only about 28 years to reach the peak level. In this respect, wage determination in SOEs is becoming more consistent not only with human capital theory, but also with other ownership sectors.

The above results indicate that the return rates of education and experience in different ownerships are different even when the selection bias is taken into consideration. In 
fact, these results are deeply related to the rigidity of Chinese labour market, where labour mobility is insufficient. Free movement between sectors is not common, so there should not be too much change after we correct for the selection bias.

\subsection{Rural-urban divide}

This section examines the wage determinations in rural and urban regions, respectively because first of all, China is a typical dual economy. The urban regions have a modern economy while the rural areas a relatively backward economy. In particular, the majority of the rural labourforce are not wage earners. And second, the reform processes was not simultaneous. China's economic reform originated in rural areas in the early 1980s, but urban reform was much later. Thus, the wage determination behaviour may be different. The results of the switching model are similar to those obtained with the OLS so only OLS results are reported. Results for 1991 are not reported either because they are similar to those of 1993.

\section{Rural regions}

In 1993, the returns to education are similar in significance, with the coefficient a little higher in the private and SCE sectors. The 1997 results are different. Education has become a critical factor in the wage determination of the private sectors, and the differentials in returns to education constitute one of the main differences in wage mechanisms of the sectors. The return to education in private sectors is 4.2 per cent (significant at the 5 per cent level), while those of other sectors remain insignificant, among which the rates of SOEs are about 1.2 per cent and of LCEs and SCEs around zero. The estimated wage-experience profiles are all inverted-U shaped in 1993. Similarly to results in previous part, wages in the private and collective-owned sectors increase faster and reach their peak levels earlier than in the SOEs, with the exception of 1997 when SOEs wages increase faster and reach the peak levels earlier.

\section{Urban regions}

The urban labour market is quite different, with the majority of the labourforce working in the public sector, especially in SOEs. The share of the private sector is extremely low, which makes our sample size very small. But the difference in wage mechanism seems obvious. The OLS results indicate that in 1993, the returns to education are all above 2 per cent, with the SOEs having the highest and only significant result. In 1997, the case is reversed to some extent, with the return rate in private sectors becoming the highest, 4.0 per cent (significant at 10 per cent level), while those of SOEs, SCEs, and LCEs' are -0.0 per cent, 1.4 per cent, and 1.7 per cent, respectively. As in rural area, the estimated wage-experience profiles are all inverted-U shaped in 1993 (except for LCE). Wages in private and collective-owned sectors increase faster and reach their peak levels earlier than the SOEs. But in 1997, it takes SOEs and private sectors nearly the same years of experience (23-25 years) to reach their peak.

Taking the results of the rural and urban areas altogether, one point is worth noting: despite the rural-urban divide, the returns to education of the different sectors in different years are very similar in pattern. The reason may be that wage earners either in the rural or urban areas are probably working in the relatively modern sectors. So too great a difference should not be expected. 
Table 6

Results for 1993 and 1997 (OLS)

Dependent variable=log (hourly wage)

\begin{tabular}{|c|c|c|c|c|c|c|c|c|}
\hline & \multicolumn{4}{|c|}{1993} & \multicolumn{4}{|c|}{1997} \\
\hline & SOEs & SCES & LCES & PRIS & SOEs & SCES & LCES & PRIS \\
\hline PANEL A & \multicolumn{8}{|c|}{ RURAL } \\
\hline Schooling & $\begin{array}{c}0.013 \\
(0.008)\end{array}$ & $\begin{array}{c}0.015 \\
(0.014)\end{array}$ & $\begin{array}{l}-0.005 \\
(0.022)\end{array}$ & $\begin{array}{c}0.018 \\
(0.025)\end{array}$ & $\begin{array}{c}0.012 \\
(0.011)\end{array}$ & $\begin{array}{l}-0.007 \\
(0.022)\end{array}$ & $\begin{array}{l}-0.005 \\
(0.029)\end{array}$ & $\begin{array}{l}0.042^{\star *} \\
(0.017)\end{array}$ \\
\hline Experience & $\begin{array}{l}0.015^{\star *} \\
(0.007)\end{array}$ & $\begin{array}{l}0.030^{\star * *} \\
(0.008)\end{array}$ & $\begin{array}{c}0.015 \\
(0.012)\end{array}$ & $\begin{array}{l}0.051^{\star \star *} \\
(0.017)\end{array}$ & $\begin{array}{l}0.034^{\star \star *} \\
(0.007)\end{array}$ & $\begin{array}{c}0.028^{\star} \\
(0.016)\end{array}$ & $\begin{array}{l}-0.006 \\
(0.026)\end{array}$ & $\begin{array}{l}0.027^{\star \star \star *} \\
(0.009)\end{array}$ \\
\hline Experience square/100 & $\begin{array}{l}-0.016 \\
(0.013)\end{array}$ & $\begin{array}{l}-0.054^{\star \star *} \\
(0.016)\end{array}$ & $\begin{array}{l}-0.028 \\
(0.022)\end{array}$ & $\begin{array}{l}-0.083^{\star * *} \\
(0.032)\end{array}$ & $\begin{array}{l}-0.057^{\star * \star} \\
(0.016)\end{array}$ & $\begin{array}{l}-0.046 \\
(0.028)\end{array}$ & $\begin{array}{c}0.032 \\
(0.060)\end{array}$ & $\begin{array}{l}-0.036^{\star \star} \\
(0.016)\end{array}$ \\
\hline Female & $\begin{array}{l}-0.062 \\
(0.056)\end{array}$ & $\begin{array}{l}-0.048 \\
(0.081)\end{array}$ & $\begin{array}{l}-0.211^{\star *} \\
(0.095)\end{array}$ & $\begin{array}{c}0.060 \\
(0.172)\end{array}$ & $\begin{array}{l}-0.079 \\
(0.049)\end{array}$ & $\begin{array}{l}-0.420^{* * *} \\
(0.108)\end{array}$ & $\begin{array}{l}-0.185 \\
(0.131)\end{array}$ & $\begin{array}{l}-0.185^{\star \star} \\
(0.086)\end{array}$ \\
\hline R2_a & 0.15 & 0.152 & 0.079 & 0.158 & 0.198 & 0.141 & 0.043 & 0.257 \\
\hline N & 367 & 308 & 168 & 232 & 469 & 224 & 117 & 225 \\
\hline PANEL B & \multicolumn{8}{|c|}{ URBAN } \\
\hline Schooling & $\begin{array}{l}0.026^{\star \star \star} \\
(0.007)\end{array}$ & $\begin{array}{c}0.023 \\
(0.022)\end{array}$ & $\begin{array}{c}0.022 \\
(0.018)\end{array}$ & $\begin{array}{c}0.023 \\
(0.036)\end{array}$ & $\begin{array}{l}-0.000 \\
(0.007)\end{array}$ & $\begin{array}{c}0.014 \\
(0.022)\end{array}$ & $\begin{array}{c}0.017 \\
(0.016)\end{array}$ & $\begin{array}{c}0.040^{*} \\
(0.021)\end{array}$ \\
\hline Experience & $\begin{array}{l}0.036^{\star \star \star} \\
(0.005)\end{array}$ & $\begin{array}{l}0.031^{* *} \\
(0.015)\end{array}$ & $\begin{array}{c}0.011 \\
(0.010)\end{array}$ & $\begin{array}{c}0.023 \\
(0.032)\end{array}$ & $\begin{array}{l}0.037^{\star \star \star} \\
(0.005)\end{array}$ & $\begin{array}{c}0.030^{*} \\
(0.016)\end{array}$ & $\begin{array}{l}0.027^{\star \star \star} \\
(0.010)\end{array}$ & $\begin{array}{l}0.041^{\text {*** }} \\
(0.011)\end{array}$ \\
\hline Experience square/100 & $\begin{array}{l}-0.040^{\star \star \star} \\
(0.009)\end{array}$ & $\begin{array}{l}-0.054^{* *} \\
(0.027)\end{array}$ & $\begin{array}{c}0.006 \\
(0.019)\end{array}$ & $\begin{array}{l}-0.031 \\
(0.055)\end{array}$ & $\begin{array}{l}-0.074^{\star * *} \\
(0.011)\end{array}$ & $\begin{array}{l}-0.068^{\star *} \\
(0.032)\end{array}$ & $\begin{array}{l}-0.040^{*} \\
(0.022)\end{array}$ & $\begin{array}{l}-0.091^{\star * \star} \\
(0.020)\end{array}$ \\
\hline Female & $\begin{array}{l}-0.015 \\
(0.045)\end{array}$ & $\begin{array}{l}-0.205^{\star} \\
(0.111)\end{array}$ & $\begin{array}{l}-0.151^{*} \\
(0.089)\end{array}$ & $\begin{array}{l}-0.563^{*} \\
(0.297)\end{array}$ & $\begin{array}{l}-0.113^{\star \star \star} \\
(0.035)\end{array}$ & $\begin{array}{l}-0.147 \\
(0.105)\end{array}$ & $\begin{array}{l}-0.132^{*} \\
(0.068)\end{array}$ & $\begin{array}{l}-0.237^{\star *} \\
(0.103)\end{array}$ \\
\hline R2_a & 0.207 & 0.368 & 0.066 & 0.383 & 0.222 & 0.126 & 0.15 & 0.436 \\
\hline $\mathrm{N}$ & 813 & 123 & 225 & 66 & 732 & 159 & 182 & 125 \\
\hline
\end{tabular}

\section{Sector choice and human capital allocation}

In this section, we look at the results obtained from the multinomial logit regression (first step of the switching model), and we expect to see the effect of education on sector choice and hence the allocation of human capital. In particular, we are interested in whether human capital is allocated to the sector where the return rates are higher. Without losing generality, we consider the 1997 results. First, let us examine the multinomial logit model, where an individuals chooses the sector based on his/her individual characteristics. We assume:

$$
\begin{aligned}
& \operatorname{Pr}(I=1 \mid z)=\frac{1}{1+\sum_{j \neq s} \exp \left(z \gamma_{j}\right)} \text {, which is the probability of choosing SOE, } 14 \text { and } \\
& \operatorname{Pr}(I=s \mid z)=\frac{\exp \left(z \gamma_{s}\right)}{1+\sum_{j \neq s} \exp \left(z \gamma_{j}\right)}
\end{aligned}
$$

14 The SOEs are considered as the base choice. 
where $s=2,3$, and 4 stands for SCE, LCE, and PRI, respectively, $z$ is a vector of independent variables defined in Section 4.1, and $\gamma$ is the coefficient vector. So we have the ratio the probability of choosing $s$ to that of choosing 1 (SOE):

$$
\begin{aligned}
& \operatorname{Pr}(I=s \mid z) / \operatorname{Pr}(I=1 \mid z)=\exp \left(z \gamma_{s}\right) \text { and we can next define relative risk ratio: } \\
& R R R \equiv \frac{\operatorname{Pr}\left(I=s \mid z^{\prime}\right) / \operatorname{Pr}\left(I=1 \mid z^{\prime}\right)}{\operatorname{Pr}(I=s \mid z) / \operatorname{Pr}(I=1 \mid z)}=\exp \left(\left(z^{\prime}-z\right) \gamma_{s}\right)
\end{aligned}
$$

in particular, when the years of schooling increased one year (holding other variables constant $), \quad R R R \equiv \exp \left(\left(z^{\prime}-z\right) \gamma_{s}\right)=\exp \left(\gamma_{\text {sshooling }}\right)$. When $R R R>1$, the ratio of the probability of choosing sector $s$ to that of choosing sector 1 increase, when $R R R<1$, the reverse is true.

Table 7 presents the results using all the available observations. Education is a significant factor in workers choosing sector (the story can also be told as follows: education is a significant factor when employers choose labour), with $R R R<1$. For example, the RRR (relative risk ratio) of SCE, LCE, and PRI are 0.81,15 0.85, and 0.79 in 1997. Casually speaking, with SOEs serving as the base choice, the above results indicate that as education increased, the relative probability of choosing other sectors decreased. This regularity contradicts the fact that the return rate of education in 1997 is lower in the SOEs, because human capital should be allocated to the sector with the higher return rates, as economic theory predicts.

Treating all available observations the same (i.e., education has the same effect on all individuals in choosing a sector) may be problematic, because different aged individuals may have different job opportunities. This consideration is indispensable in view of the fact that China has experienced remarkable change over the past two decades. We divide the whole sample into five subgroups according to age: $<=25,25 \sim 30,30 \sim 35,35 \sim 40$,

Table 7

Ownership choice model (1997, multinomial logit)

\begin{tabular}{|c|c|c|c|c|c|c|c|c|c|}
\hline & & \multicolumn{2}{|c|}{ SCES/SOEs } & \multicolumn{2}{|c|}{ LCEs/SOEs } & \multicolumn{2}{|c|}{ PRI/SOES } & \multirow[b]{2}{*}{ Ps R2 } & \multirow[b]{2}{*}{ Obs } \\
\hline & & Beta & RRR & Beta & RRR & Beta & RRR & & \\
\hline A: & Full sample & $-0.2053^{\star \star \star}$ & 0.8144 & $-0.1610^{\star \star \star}$ & 0.8513 & $-0.2356^{\star \star \star}$ & 0.7901 & 0.2318 & 2176 \\
\hline \multirow[t]{5}{*}{ B: } & $<=25$ & $-0.5580^{\star \star \star}$ & 0.5724 & -0.1973 & 0.821 & $-0.4948^{\star \star \star}$ & 0.6097 & 0.3167 & 345 \\
\hline & $25-30$ & -0.3168 & 0.7285 & -0.1651 & 0.8478 & $-0.5429 \star \star$ & 0.581 & 0.2943 & 318 \\
\hline & $30-35$ & $-0.3644^{\star \star}$ & 0.6946 & 0.0763 & 1.0793 & -0.1589 & 0.8531 & 0.3172 & 373 \\
\hline & $35-40$ & 0.0262 & 1.0265 & 0.1673 & 1.1821 & 0.0502 & 1.0515 & 0.4591 & 308 \\
\hline & $>40$ & $-0.2138^{\star \star *}$ & 0.8075 & $-0.2267^{\star \star \star}$ & 0.7971 & $-0.2940^{\star \star \star}$ & 0.7453 & 0.2618 & 829 \\
\hline
\end{tabular}

Dependent variable=ownership $(\mathrm{SOE}=1 / \mathrm{SCE}=2 / \mathrm{LCE}=3 / \mathrm{PRI}=4)$

Note: We also controlled for experience, experience square, family size, gender ratio, marital status, urban dummy, family asset, etc., but due to space limitations, are not reported here. Constant and standard errors also not reported. * Significant at $10 \%$ level, ** significant $5 \%$ level,

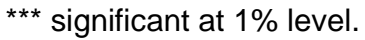

15 Assume when the years of schooling are $a, \operatorname{Prob}_{a}(\mathrm{PRI}) / \operatorname{Prob}_{a}(\mathrm{SOE})=r$, then when years of schooling are $a+1$ Prob $_{a+1}(\mathrm{PRI}) / \operatorname{Prob}_{a+1}(\mathrm{SOE})=0.811 r$. 
and above 40. The results are reported in Panel B of Table 7. The results are similar to Panel A: the more-educated labour education tend to choose the SOEs. For those younger than 25 years old, the RRR of SCEs, LCEs, and PRIs relative to SOEs are 0.57 (significant at 1 per cent level), 0.82 and 0.61 (significant at 1 per cent level). The differences in magnitude and significance between PRIs-SCEs and LCEs indicate that the SOEs and LCEs may basically be more familiar, and this may also be true for the SCE and PRI sectors. The only one exception is the group of 35-40 year olds, for whom education is not a significant factor in the sector choice. Also, those with more years of schooling tend to choose the non-state sector $(R R R>1)$.

Briefly, individuals with more human capital tend to be allocated to the SOE sectors even though the return to education is lower. This may be explained by the perception that in the SOEs sectors, job are more decent, employees face fewer unemployment risks, and the fringe benefits constitute a great part of the employees' income.

\section{Conclusion}

This paper aims to investigate the wage mechanisms in sectors that are state-owned, collective-owned, and private. Using CHNS data, we estimate the return to education and find that the return rates are different in the various sectors. This finding seems to be robust when we consider selection bias and the rural-urban divide. One important finding is that private-sector return rates to education are the highest in 1997, which indicates that the private sector compensates human capital more in terms of wages. We tend to conclude that China's labour market is segmented at least in terms of wage determination.

This paper may also have some policy implications. The fact that labour with more human capital tend to choose the state sector where the returns are lower indicates that the nature of the job may be different between the state- and non-state sectors. One possible reason may be the lack of such fringe benefits as social security in the private sectors. The case of state sector may be reversed when more weight is given to nonwage compensation and the labourforce may lack sufficient mobility. There is sufficiently large space for a benevolent and efficient government to perform its duty.

In sum, estimating wage equations is only one method of understanding China's labour market, but It is only the beginning in understanding more complicated and challenging issues such as income distribution and inequality. This research study highlights that we need to give greater attention to the ownership structure of China's economy, which is essential for a transitional economy. 


\section{References}

Appleton, S., L. Song, and Q. Xia (2005). 'Has China Crossed the River? The Evolution of Wage Structure in Urban China during Reform and Retrenchment'. Journal of Comparative Economics, 33 (4): 644-63.

Becker, G. S. (1993). Human Capital: A Theoretical and Empirical Analysis with Special Reference to Education, 3rd edn. Chicago and London: University of Chicago Press.

Card, D. (1999). 'Causal Effects of Education on Earnings', in O. Ashenfelter and D. Card (eds), Handbook of Labour Economics. Amsterdam: Elsevier.

Chen, Y., S. Demurger, and M. Fournier (2005). 'Earnings Differentials and Ownership Structure in Chinese Enterprises’. Economic Development and Cultural Change, 53 (4): 933-58.

Chen, Y., and X. Chunbing (2004). 'Rural Modernization and the Role of Education in Rural Labour Market’. Economic Research Journal (Jingji Yanjiu), August. In Chinese.

de Brauw, A., J. Huang, S. Rozelle, L. Zhang, and Y. Zhang (2002). 'The Evolution of China's Rural Labour Markets during the Reforms: Rapid, Accelerating, Transforming'. Journal of Comparative Economics, 30 (2): 329-53.

Dong, X., and P. Bowles (2002). 'Segmentation and Discrimination in China's Emerging Industrial Labour Market’. China Economic Review, 13 (2-3): 170-96.

Duflo, E. (2001). 'Schooling and Labour Market Consequences of School Construction in Indonesia: Evidence from an Unusual Policy Experiment'. American Economic Review, 91 (4): 795-813.

Duncan, T., and J. Strauss (1997). 'Health and Wages: Evidence on Men and Women in Urban Brazil’. Journal of Econometrics, 77: 159-85.

Fogel, R. W. (1994). 'Economic Growth, Population Theory, and Physiology: The Bearing of Long-Term Processes on the Making of Economic Policy'. American Economic Review, 84 (3): 369-95.

Groves, T., Y. Hong, J. McMillan, and B. Naughton (1995). 'China's Evolving Managerial Labour Market’. The Journal of Political Economy, 103 (4): 873-92.

Gyourk, J., and J. Tracy (1988). 'An Analysis of Public- and Private-Wages Allowing for Endogenous Choices of Both Government and Union Status'. Journal of Labour Economics, 6 (2): 229-53.

Hay, J. (1980). 'Selectivity Bias in a Simultaneous Logit-OLS Model: Physician Specialty Choice and Specialty Income'. Farmington: University of Connecticut Health Center. Unpublished manuscript.

Johnson, E. N., and G. C. Chow (1997). 'Rates of Return to Schooling in China'. Pacific Economic Review, 2 (2): 101-13.

Lardy, N. (1997). China's Unfinished Economic Reform. Washington, DC: Brookings Institution Press. 
Lee, L. F. (1978). 'Unionism and Wage Rates: A Simultaneous Equation Model with Qualitative and Limited Dependent Variables’. International Economic Review, 19 (2): 415-33.

Li, S. (1997). 'A Model of Labour Mobility in Transition China'. Economic Research Journal (Jingji Yanjiu), January. In Chinese.

Liu, Z. (1998). 'Earnings, Education and Economic Reforms in Urban China'. Economic Development and Culture Change, 46 (4): 697-725.

Maddala, G. S. (1983). Limited-dependent and Qualitative Variables in Econometrics. Cambridge: Cambridge University Press.

Maurer-Fazio, M. (1999). 'Earnings and Education in China's Transition to a Market Economy Survey Evidence from 1989 and 1992’. China Economic Review, 10 (1): 17-40.

Meng, X. (1996). 'An Examination of Wage Determination in China's Rural Industrial Sector'. Applied Economics, 28 (1): 715-24.

Meng, X. (2000). Labour Market Reform in China. Cambridge: Cambridge University Press.

Mincer, J. (1974). Schooling, Experience and Earnings. New York: Columbia University Press.

NBS (National Bureau of Statistics) (1997). China Statistical Yearbook. Beijing: China Statistic Publishing House. Available at: www.stats.gov.cn/ndsj/information/nj97/ dml.htm .

Parish, W. L., X. Zhe, and F. Li (1995). 'Nonfarm Work and Marketization of the Chinese Countryside’. China Quarterly, 143 (Sept): 697-730.

Psacharopoulos, G. (1994). 'Returns to Investment in Education: a Global Up-Date'. World Development, 22: 1325-43.

Robinson, C., and N. Tomes (1984). 'Union Wage Differentials in the Public and Private Sectors: A Simultaneous Equations Specification'. Journal of Labour Economics, 2 (1): 106-27.

Schultz, T. W. (1961). 'Investment in Human Capital'. American Economic Review, 1 (2): $1-17$.

Strauss, J., and D. Thomas (1998). 'Health, Nutrition and Economic Development'. Journal of Economic Literature, 36 (2): 766-817.

Trost, R. P. (1977). 'Demand for Housing: A Model Based on Inter-related Choices Between Owning and Renting’. Jacksonville: University of Florida. PhD thesis.

Wei, Z. (2004). 'The Role of Health on Off-farm Employment and Wage Decision'. Economic Research Journal (Jingji Yanjiu), February: 64-74. In Chinese.

Wooldridge, J. M. (2002). Econometric Analysis of Cross Section and Panel Data. Cambridge, MA: MIT Press.

Zhang, J., Y. Zhao, A. Park, and X. Song (2005). 'Economic Returns to Schooling in Urban China, 1988 to 2001'. Journal of Comparative Economics, 33 (4): 730-52. 
Zhang, L., J. Huang, and S. Rozelle (2002). 'Employment, Emerging Labour Market, and the Role of Education in Rural China'. China Economic Review, 13 (2-3): 313-28.

Zhao, Y. (2001). 'Foreign Direct Investment and Relative Wages: The Case of China'. China Economic Review, 12 (1): 40-57.

Zhao, Y. (2002). 'Earnings Differentials between State and Non-State Enterprises in Urban China’. Pacific Economic Review, 7 (1): 181-97. 